\title{
NUEVOS DATOS SOBRE LA PRIMERA METALURGIA DEL BRONCE EN EL NOROESTE DE LA PENÍNSULA IBÉRICA: LA CONTRIBUCIÓN DE BOUÇA DA COVA DA MOURA (ARDEGÃES, MAIA, PORTUGAL)*
}

\author{
BeAtriz Comendador ReY(1) \& AnA M. S. BetTenCOURT ${ }^{(2)}$
}

Resumen

Abstract

\begin{abstract}
A propósito del estudio de un resto de fundición de bronce procedente del lugar de Bouça da Cova da Moura (Ardegães, Maia), a incluir en el conjunto de bronces de mayor antigüedad del noroeste peninsular, efectuaremos una revisión sobre la caracterización de las primeras aleaciones de bronce de esta región, con base en la interpretación de los resultados arqueometalúrgicos obtenidos en los últimos años. A este propósito se referirán los nuevos resultados de las aplicaciones de la Fuente de Radiación Sincrotrónica (Synchrotron Radiation Source - SRS) y de la Fuente de Dispersión de Neutrones (Neutron Spallation Source - ISIS), así como su importancia en la elaboración de nuevas hipótesis sobre la primera metalurgia del bronce del noroeste peninsular.
\end{abstract}

Palabras Clave: Arqueometalurgia; Bronce; Bronce Medio; Noroeste Peninsular.

\begin{abstract}
New data on the first tin metallurgy in the Northwest of the Iberian Peninsula: the contribution of Cova da Bouça da Moura (Ardegães, Maia, Portugal)

This text presents an overview of the characterization of the first bronze alloys in the Northwest of the Iberian Peninsula, from a study of a bronze droplet from Bouca Cova da Moura (Ardegão, Maia, North of Portugal). This was based on the interpretation of archaeometallurgical results obtained in recent years. The study references new results from the application of Synchrotron Radiation Source (SRS) and Source Neutron Scattering (Spallation Neutron Source - ISIS) as well as its importance in developing new hypotheses about the first bronze metallurgy of the Northwest of the Iberian Peninsula.
\end{abstract}

Key-words: Archaeometallurgy; Bronze; Middle Bronze Age; Northwest of Iberian Peninsula.

\section{Bouça da Cova da Moura (ARDegães, MAIA)}

El lugar de Bouça da Cova da Moura se localiza en Ardegães, feligresía de Águas Santas, ayuntamiento de Maia, distrito de Porto, Norte de Portugal, en el curso medio de la bacía del rio Leça (Figs. 1 y 3.3), a unos 13-14 Km de yacimientos primarios de estaño, según la Carta Geológica de Portugal 9C (Penafiel), escala 1:50.000. Las excavaciones arqueoló- gicas realizadas en el lugar, revelaron la existencia de deposiciones de la Edad del Bronce en el contexto de un área arqueológica más amplia, donde igualmente aparecen monumentos megalíticos, un amplio núcleo de grabados rupestres (de donde fue extraída la famosa "Pedra de Ardegães") y materiales de superficie atribuibles al Calcolítico y a la Edad del Bronce (BETTENCOURT et al. 20071; Ribeiro et al. 2010; BETTENCOURT 2010b).

A pesar de que los sondeos arqueológicos, rea-

* Este texto resulta de una comunicación presentada en las I Jornadas Arqueológicas da Bacia do Rio Leça, Matosinhos, em Outubro de 2007. La presentación en power point fue publicada, desde 2007, on-line en la URL: http://webs.uvigo.es/beacomendador/index_archivos/Jornadas.pps

(1) Área de Prehistoria, Departamento de Historia, Arte e Xeografía. Facultade de Historia de Ourense. Campus das Lagoas, 32004, Ourense. Investigadora del Grupo GEAAT de la Universidade de Vigo e Investigadora Asociada del Centro de Investigação Transdisciplinar Cultura, Espaço e Memória - CITCEM. E-mail: beacomendador@uvigo.es

(2) Departamento de História, Instituto de Ciências Sociais, Campus de Gualtar, Universidade do Minho, 4710-057 Braga, Portugal. Investigadora do Centro de Investigação Transdisciplinar Cultura, Espaço e Memória - CITCEM, financiado por Fundos Nacionais através da FCT - Fundação para a Ciência e a Tecnologia no âmbito do projecto PEst-OE/HIS/UI4059/2011. E-mail: anabett@uaum. uminho.pt

1 A.M.S. Bettencourt, L.B. Alves, A.T. Ribeiro \& R.T. Menezes. O lugar da Bouça da Cova da Moura (Ardegães, Maia), no contexto da Pré-História do vale do Leça. Comunicação apresentada oralmente a las I Jornadas Arqueológicas da Bacia do Rio Leça, Matosinhos, Outubro de 2007. 


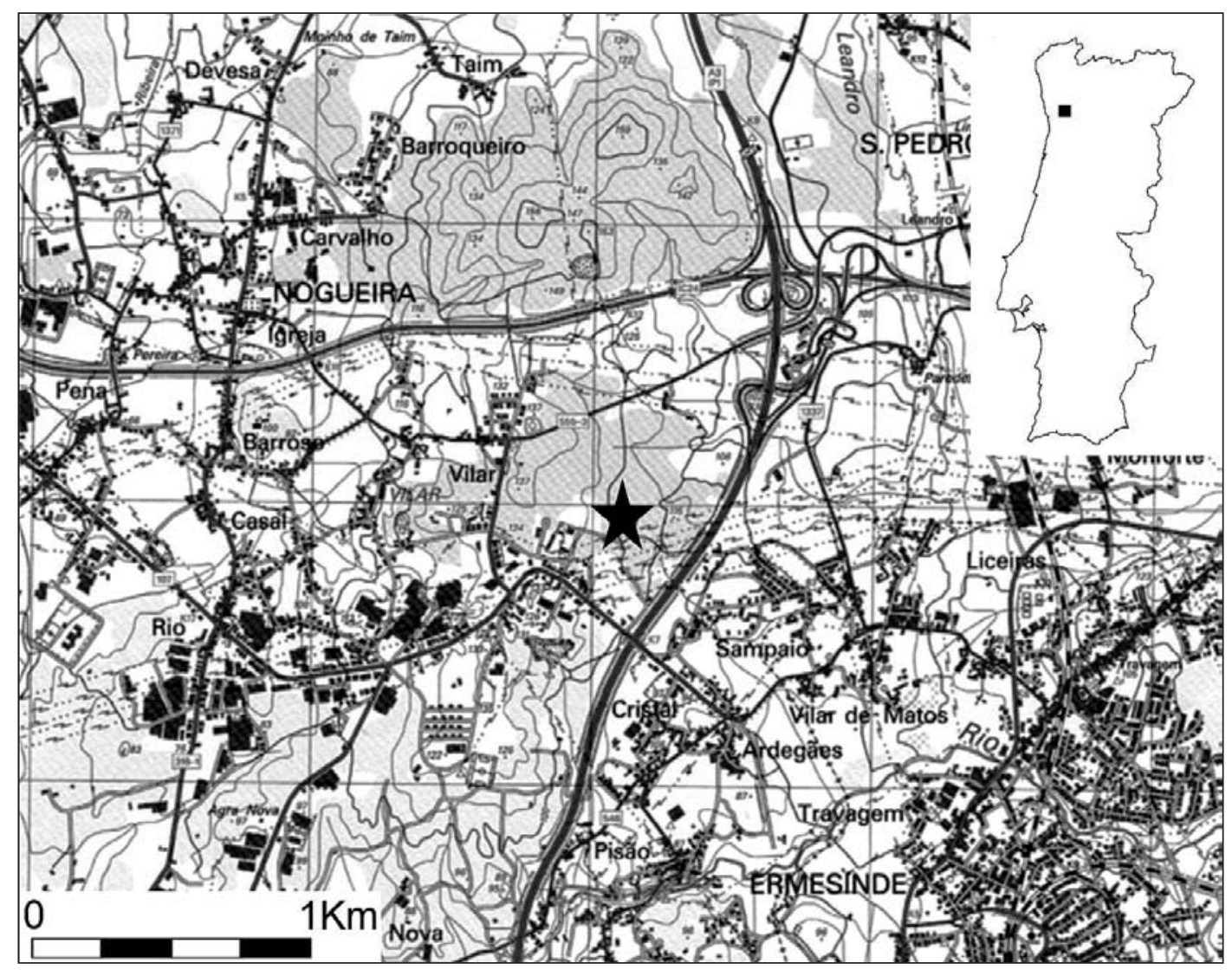

Fig. 1. Ubicación del hallazgo de la gota de fundición de bronce en Bouça da Cova da Moura (Ardegães, Maia) en la Carta Militar de Portugal, n. ${ }^{0}$ 110, esc. 1: 25000.

Fig. 1. Location of the bronze droplet from Cova da Moura da Bouça (Ardegães, Maia) in the Military Map of Portugal, n. ${ }^{\circ}$ 110, scale. 1: 25000 (IGEOE).

lizados en Marzo de 2004, incidieron sobre un área muy pequeña y ya bastante alterada por la plantación de eucaliptos (sector 2), fue posible poner al descubierto negativos de estructuras en materiales perecederos, materializadas por agujeros de poste, algunos bien delimitados por piedras, y restos de arcilla compactada pertenecientes presumiblemente a un pavimento. En este contexto se recogieron numerosos fragmentos cerámicos de fabricación manual, cocciones reductoras y pastas arenosas, entre los que identificamos varias formas de recipientes, tales como un vaso troncocónico, un vaso de "medio bordo horizontal"'2 liso, así como un número significativo de decoraciones plásticas, en forma de cordones, que permiten insertar esta ocupación en el Bronce Medio del noroeste de Portugal. Fue en este contexto general que se exhumó una gota de fundición fragmentada en dos partes, en el cuadrado D4 (Fig. 2). Esta presentaba una tonalidad verde oscura en la superficie externa y verde más clara

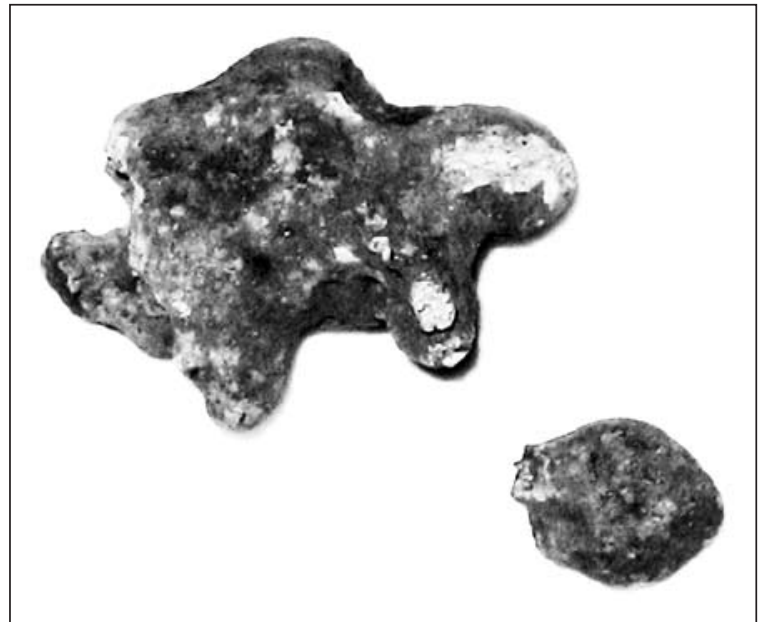

Fig. 2. Gota de fundición de Bouça da Cova da Moura (Ardegães, Maia). (Fragmento mayor: $22 \mathrm{~mm}$ de largo).

Fig. 2. Bronze droplet from Cova da Moura Bouça (Ardegães, Maia). (Biggest fragment: $22 \mathrm{~mm}$ long). 
en la interna. Los fragmentos tenían las siguientes dimensiones:

Fragmento 1:

Largo - $22 \mathrm{~mm}$; Ancho - $18 \mathrm{~mm}$; Peso - $5 \mathrm{~g}$

Fragmento 2:

Largo - $9 \mathrm{~mm}$; Ancho $-7 \mathrm{~mm}$; Peso $-<1 \mathrm{~g}$

Tras su recogida no se le realizó ningún tratamiento de limpieza, consolidación o estabilización.

\section{Bouça da Cova da Moura en el Contexto de los Primeros Bronces Del Noroeste Peningular}

La aleación bronce aparece en el noroeste peninsular a inicios del II milenio AC, en una pequeña escala de producción, distribución y consumo (Fig. 3).

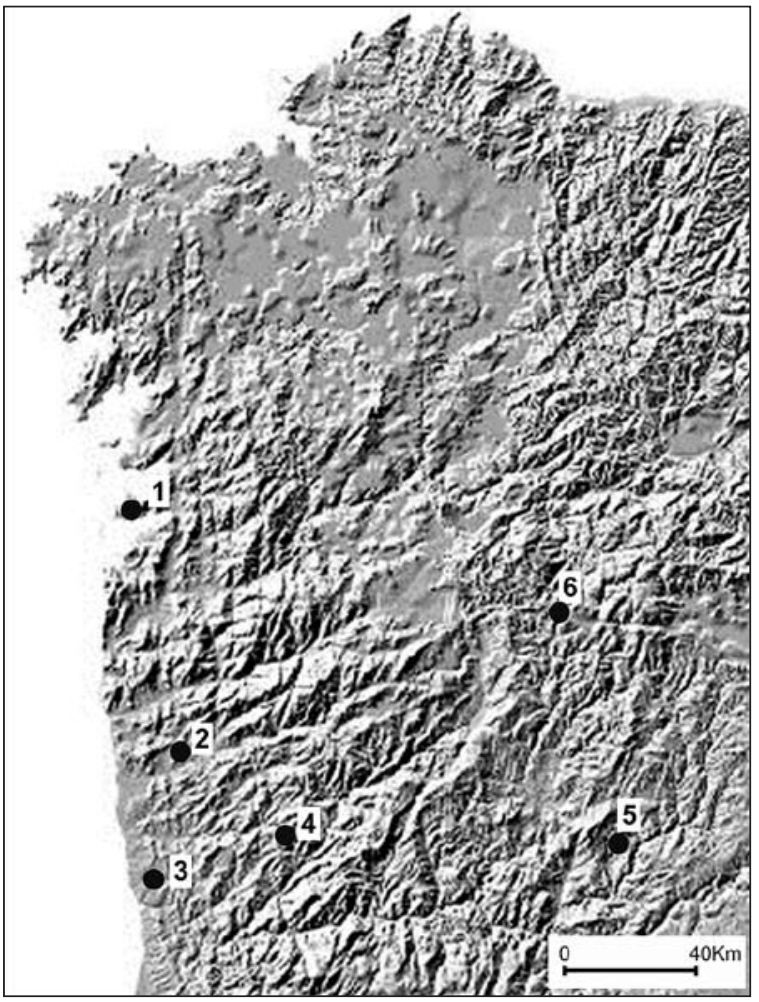

Fig. 3. Yacimientos con evidencias tempranas de producción de bronce en el contexto del noroeste peninsular: 1 - Fixón - Costa da Seixeira (Cangas); 2 - Sola (Braga); 3 - Bouça da Cova da Moura, Ardegães (Maia); 4 - Cimalha (Felgueiras); 5 - Fraga dos Corvos (Macedo de Cavaleiros); 6 - Erosa (A Gudiña).

Fig. 3. Archaeological sites with early evidence of bronze production in the Northwestern Iberian Peninsula: 1 - Fixón - Costa da Seixeira (Cangas); 2 - Sola (Braga); 3 - Cova da Moura Bouça, Ardegães (Maia); 4 - Cimalha (Felgueiras); 5 - Fraga dos Corvos (Macedo de Cavaleiros); 6 - Erosa (A Gudiña).
El primer contexto con datos sobre la práctica de metalurgia del bronce es el nivel de ocupación IIb del poblado de A Sola (Braga) (Fig. 3.2). Además de una gota y un resto de fundición, se detectó un posible fragmento de barra o varilla en bronce, algunos crisoles de pequeñas dimensiones, un presumible molde para la fundición de hachas tipo "Bujões-Barcelos" (Fig. 4), restos de corneanas no utilizadas, pero, posiblemente, seleccionadas para la reducción de cobre, y fosas con canales probablemente para actividad metalúrgica (Fig. 5). Todo este conjunto de materiales fue datado por radiocarbono de entre inicios del siglo XVII y los finales de XVI AC (BETTENCOURT 1999, 2000, 2001; BETTENCOURT \& COMENDADOR 2004)

Otro sitio extremadamente significativo en este contexto es el de Fraga dos Corvos, en Macedo de Cavaleiros, distrito de Bragança (Fig. 3.5). Allí fue

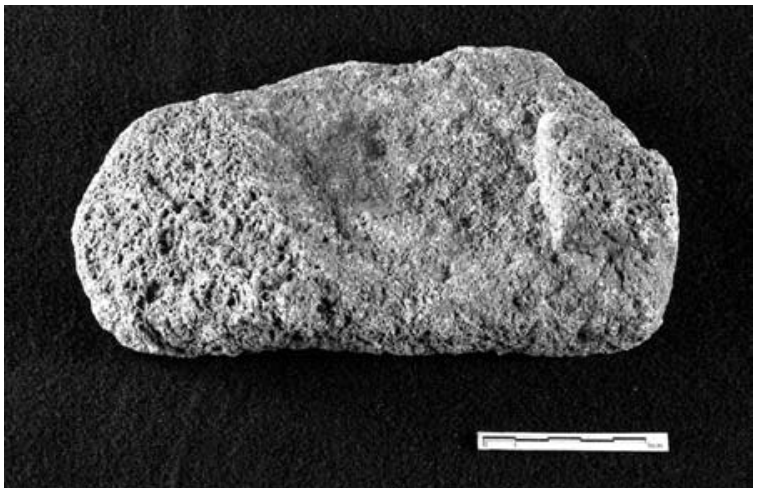

Fig. 4. Posible molde para la fundición de hachas tipo "Bujões-Barcelos" de A Sola IIb (Foto MDDS / / Manuel Santos).

Fig. 4. Probable axe casting mold of the "Bujōes-Barcelos" type from Sola IIb (Photo: MDDS / Manuel Santos).

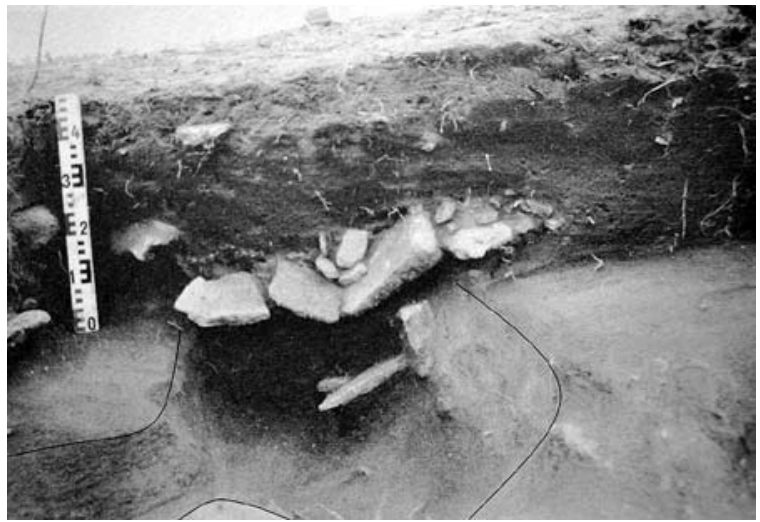

Fig. 5. Fosa N. ${ }^{\circ}$ 9, con canal lateral, probablemente para actividad metalúrgica, en A Sola IIb (BETTENCOURT 2000).

Fig. 5. Pit N. ${ }^{\circ} 9$, with side channel, probably for metallurgical activity, found at Sola IIb (BETTENCOURT 2000). 
detectada una estructura registrada como el número 6 e interpretada como cabaña o alpendre, en el interior de la cual existía un horno u hogar para el calentamiento de los crisoles y la fusión del metal. A la par que esta estructura, apareció otra identificada como cabaña 4, provista de una caja de arena y que serviría para el vertido del metal fundido en el interior de los moldes. Entre estos dos espacios, es decir, entre el hogar y la caja de arena, se exhumaron gotas de fundición y elementos vitrificados no metá$\operatorname{licos}^{3}$. Las cerámicas asociadas incluyen recipientes tipo Cogeces e Protocogotas (SENNA-MARTínez et al. 2005, 2006; SENNA-MARTíneZ \& LUís 2009), por lo que se atribuye al conjunto una cronología del Bronce Medio.

También en el gran poblado de Cimalha, Felgueiras, distrito de Porto (Fig. 3.4), en contexto de excavación arqueológica, apareció un molde doble de hachas de tipo "Bujões-Barcelos", aún inédito 4 , lo que revela indicios de producción metalúrgica del Bronce Medio en ese lugar.

En el yacimiento de O Fixón - A Costa da Seixeira, Cangas, Pontevedra (Fig. 3.1), fue detectada una gota de fundición aparentemente en asociación con cerámicas campaniformes, campaniformes evolucionados y formas cerradas con profusión de decoración plástica a base de cordones, así como "bordes revirados" o con tendencia a horizontales que permitieron su atribución al Bronce Inicial, según J. Suárez (1995). De cualquier forma, la falta de información estratigráfica sobre este yacimiento aconseja prudencia en cuanto a la atribución cronológica propuesta, pudiendo ser algunos materiales eventualmente de un Bronce Medio, dadas las cronologías conocidas para estos vasos en el noroeste portugués.

Bouça da Cova da Moura, en Maia, es pues el quinto lugar con evidencias de producción de bronce en el contexto en todo el noroeste peninsular.

Además de los sitios referidos, que parecen ser lugares de producción in situ, hay otros contextos de hallazgos ya relacionados con prácticas funerarias, depósitos o minas, ya descontextualizados, que testimonian de forma indirecta la práctica de la primera metalurgia del bronce en este período cronológico-cultural.

En términos de los registrados en contextos funerarios o en sus inmediaciones, referimos el hacha plana asociada a un brazal de arquero del túmulo de los Fitos, Llena (BLAS 1983), el brazalete del lugar de A Lagoa, Corvilho, Santo Tirso, asociado a vasos troncocónicos, a un vaso subcilíndrico y a "largos bordos horizontais" (SANCHES 1982; CASTRO PÉrEZ 1997) (Fig. 6), todos ellos con vestigios de hollín parcial en el interior y exterior, indicando su

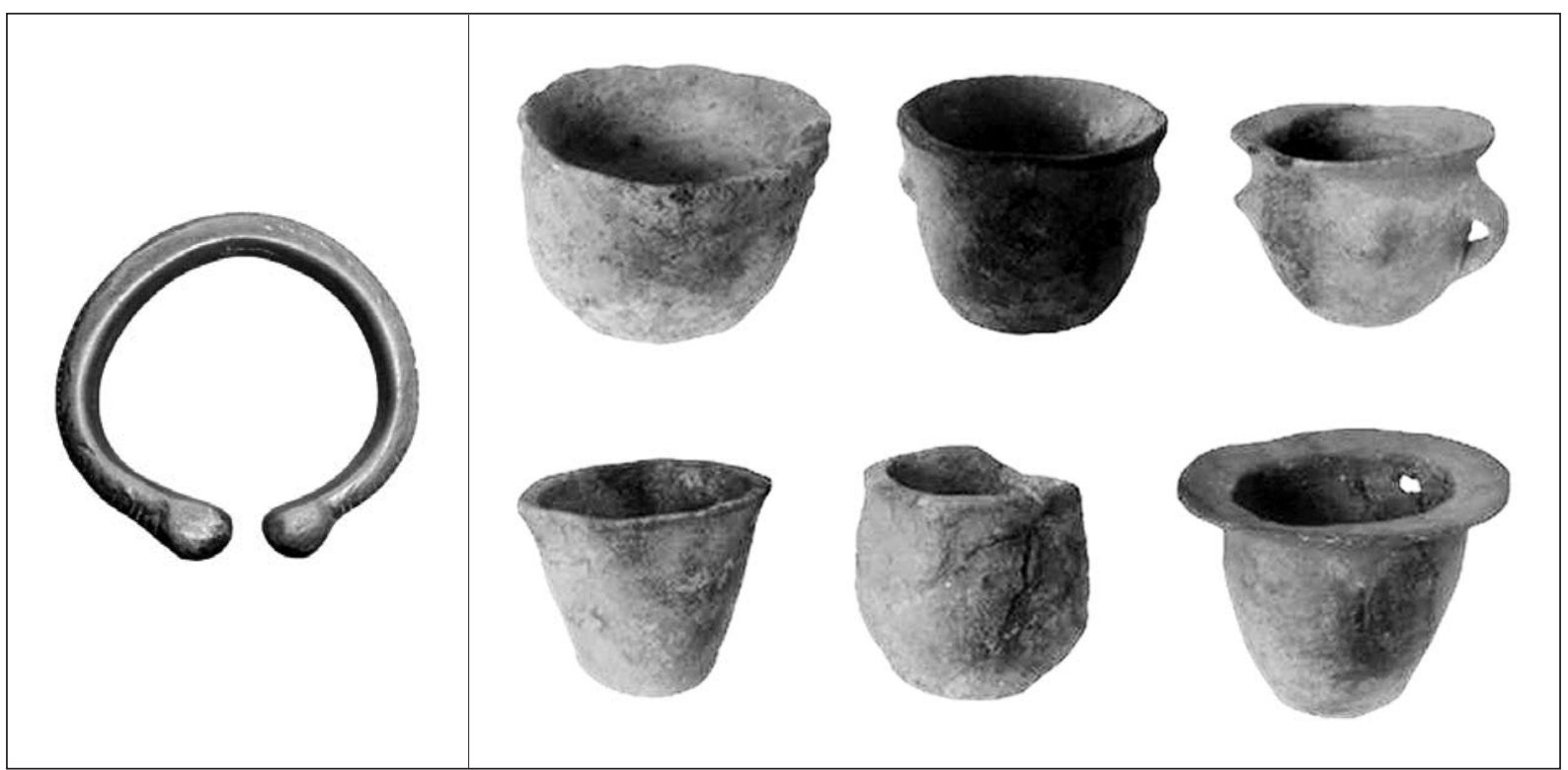

Fig. 6. Brazalete (61 cm de diámetro interno) y vasos del lugar de A Lagoa (Corvilho, Santo Tirso). (Fotos: Cámara Municipal Sto. Tirso).

Fig. 6. Bracelet (61 cm internal diameter) and vessels from Lagoa (Corvilho, Santo Tirso). (Photos: City Council of Sto. Tirso).

Por aquel entonces, se consideraron escorias, aunque análisis posteriores demostraron que no son tal (GEIRINHAS et al. 2011).

Amablemente mostrado por Andreia Arezes, directora de las excavaciones realizadas en la vertiente Oeste de Cimalhas, al servicio de la empresa de arqueología Perennia Monumenta, Serviços Técnicos de Arqueologia, Lda. 
utilización funeraria y una muy probable cronología del Bronce Medio (BetTencourt 2010) y, eventualmente, los punzones asociados espacialmente a una posible estructura funeraria del islote de Guidoiro Areoso, Illa de Arousa (COMENDADOR 1998) donde hay deposiciones del Calcolítico y del Bronce Inicial y Medio, atendiendo a los paralelos gallegos y portugueses para las cerámicas encontradas 5 y la datación por radiocarbono GrN-16108 4020 $440 \mathrm{BP}$ sobre concha, que según la calibración a 2 sigma de F. Alonso y J.M. ${ }^{a}$ Bello (1997) situaría una de las reutilizaciones de ese espacio entre 1773-1536. Una nueva calibración de esta datación realizada en Laboratório de Radiocarbono do ITN en Sacavém, la coloca entre 2413-1802 $\mathrm{AC}^{6}$, es decir, en el Bronce Inicial (Fig. 7).

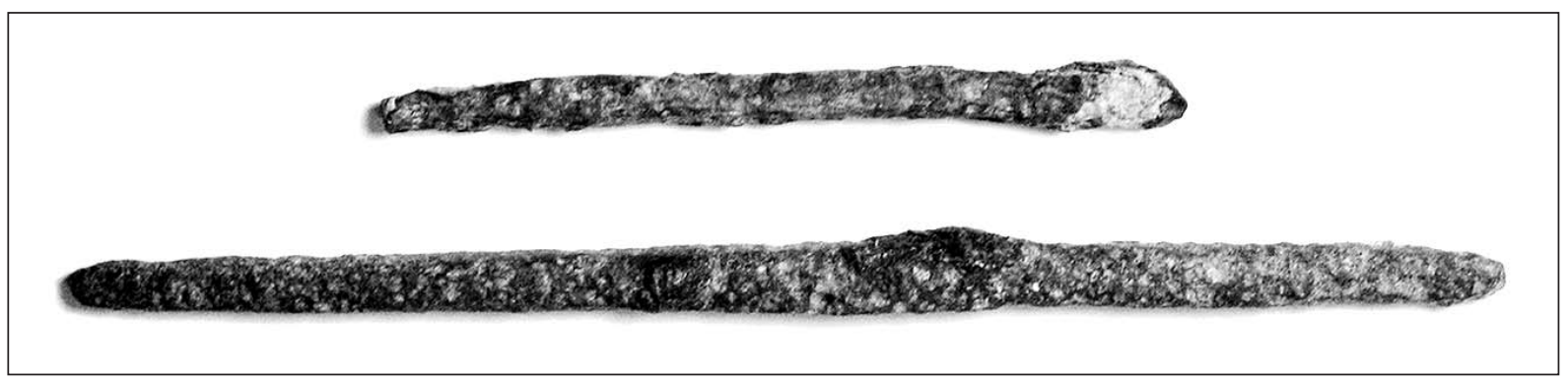

Fig. 7. Punzones de Guidoiro Areoso (Illa de Arousa, Pontevedra). (El punzón más largo mide $112 \mathrm{~mm}$ de largo).

Fig. 7. Awls of Guidoiro Areoso (Illa de Arousa, Pontevedra). (The biggest awl is $112 \mathrm{~mm}$ long).

En relación a los depósitos destacamos el de A Cova da Bouça (São Bartolomeu do Mar, Esposende, Braga), compuesto por un hacha plana, otra de tipo "Bujões-Barcelos" (Fig. 8.1) y por una espada de cobre de corona de remaches (ALMEIDA 1988); el de Agro Velho (Montalegre, Vila Real), con cinco hachas de tipo "Bujões-Barcelos" (TEIXEIRA \& FERNANDES 1963-1964); el del Lugar da Mata / S. João do Rei (Póvoa de Lanhoso, Braga) con cerca de treinta hachas planas de tipo "Bujões-Barcelos" (Cortez 1946; BetTenCourt 1999, 2001) (Fig. 8.2); el del Monte de St. ${ }^{\circ}$ Ovídio (Ponte de Lima, Braga) donde, de hecho, aparecieron apenas dos hachas, una de ellas cortada y otra de tipo "Bujões-Barcelos"7 (CORTEZ 1946) (Fig. 8.3). En Galicia hay que destacar el depósito de un hacha plana, de un hacha de tipo "Bujões-Barcelos" y de dos puñales en Codeseda (A Estrada, Pontevedra) (Fig. 9) así como el depósito de depósito de Outeiro (Sta. M. ${ }^{\mathrm{a}}$ de Campos, Melide, A Coruña) ${ }^{8}$.

En relación con minas, aunque de contexto impreciso, conocemos las hachas de tipo "Bujões-Barcelos" encontradas en Sta. Mariña de Anxeriz, Tordoia (COMENDADOR 2007) (Fig. 10.1) (Fig. 3.6) y en la mina Folgadoura, Viana do Castelo (ZBYZEWSKI \& FERREIRA 1955) (Fig. 10.2).

Por último hay que mencionar los hallazgos de moldes de hachas en piedra, descontextualizados de Erosa (A Gudiña, Ourense) (Monteagudo 1977: n. ${ }^{\circ}$ 735AB) y del Monte das Carballas (Guillade, Ponteareas, Pontevedra) (SUAREZ et al. 1997).

5 Las cerámicas con incisiones metopadas de tipo Penha, muy comunes en la primera fase de Guidoiro, son conocidas desde el Neolítico Final en la cuenca del Támega, en Portugal, aunque en asociación con otras matrices decorativas. Su casi exclusividad parece ser una característica del Calcolítico, es decir, de los finales del IV al $3 .^{\circ}$ cuarto del III milenio AC, momentos para los que existen diversas dataciones radiocarbónicas. Ver a propósito S. Jorge (1986), A.M.S. Bettencourt et al. (2007); R. Fábregas et al. (2007) entre otros y datos inéditos. Los vasos de largo bordo horizontal, típicos de la última fase de Guidoiro, están fechados, por datas de radiocarbono, en el Noroeste portugués de entre el Bronze Médio (BETTEnCOURT 1997, 1999, 2000) al Bronze Final (JoRGE 1988, BETTENCOURT 1997).

6 Agradecemos a António Monge Soares la calibración de esta datación. Se utilizó para calibración la curva Marine09 (REIMER et al. 2009) y lo programa CALIB 6.0.1 (STUIVER \& REIMER 1993).

7 La identificación de un lingote plano-convexo depositado entre las colecciones del antiguo Museo de Etnología do Porto (BETTENCOURT \& COMENDADOR 2004: nota 11) como procedente de St. ${ }^{\circ}$ Ovídio, corresponde, ciertamente, a un error, pues según deducimos posteriormente, a través de las descripciones de L. Monteagudo (1954) y de A. Coffyn (1995), ese lingote proviene del depósito de la Quinta da Fonte Velha (Viatodos, Barcelos), ya del Bronce Final o del Hierro Inicial (BetTENCOURT 2001).

8 Para una revisión de los depósitos gallegos con hachas así como hallazgos aislados consultar B. Comendador (1999b) y A.M.S. Bettencourt y B. Comendador (2004). 


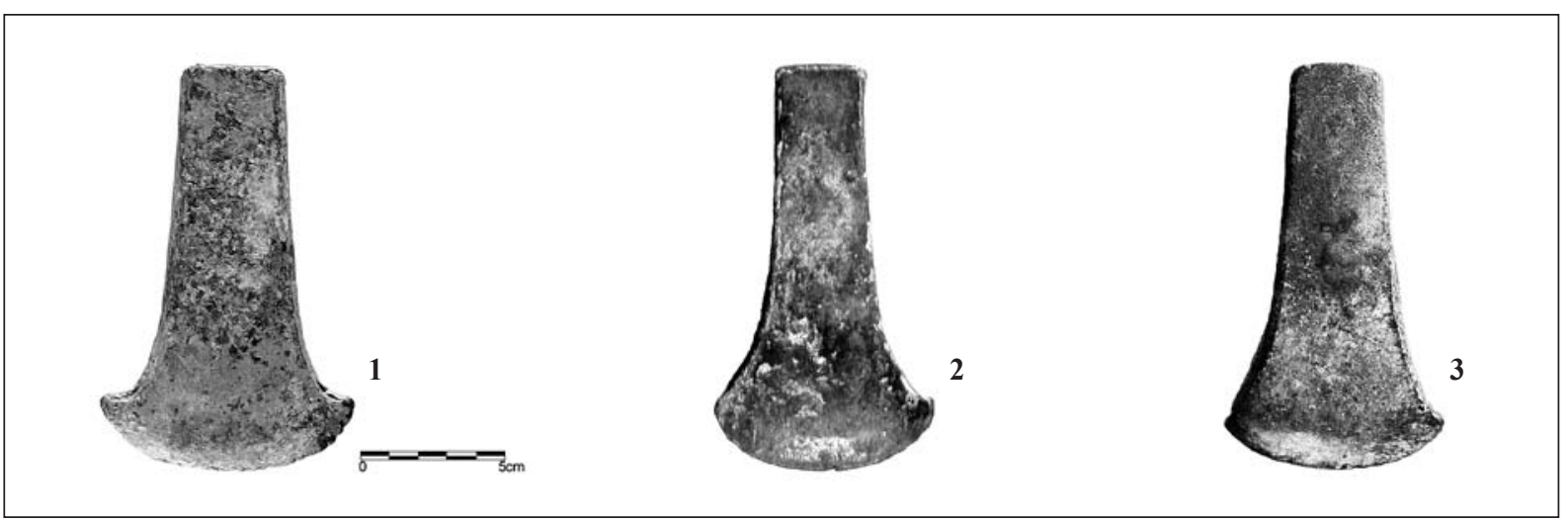

Fig. 8. 1 - Una de las hachas del depósito de Cova da Bouça (São Bartolomeu do Mar, Esposende, Braga). (Foto MDDS / / Manuel Santos). 2 - Hacha del depósito de Lugar da Mata (?) / S. João do Rei (Póvoa de Lanhoso, Braga). (Mede de largo $143 \mathrm{~mm}$ ). 3 - Hacha del depósito de Monte de St. ${ }^{\circ}$ Ovídio (Ponte de Lima, Braga). (Mede de largo $156 \mathrm{~mm}$ ).

Fig. 8. 1 - One of the axes of the Cova da Bouça (São Bartolomeu do Mar, Esposende, Braga) metal hoard (Photo: MDDS / Manuel Santos). 2 - Axe from the metal hoard of Lugar da Mata / S. João do Rei (Póvoa de Lanhoso, Braga). (The axe is $143 \mathrm{~mm}$ long). 3 - Axe from the metal hoard of Monte St. ${ }^{\circ}$ Ovídio (Ponte de Lima, Braga). (The axe is $156 \mathrm{~mm}$ long).

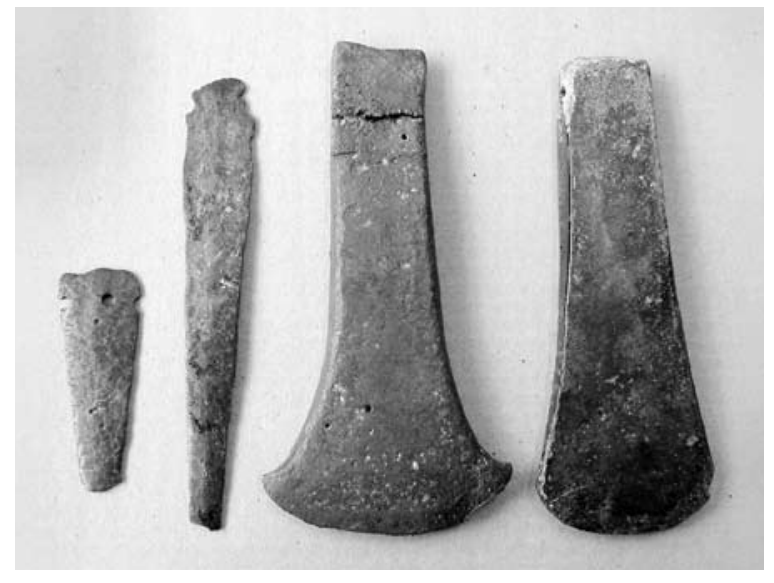

Fig. 9. Depósito de Codesesa (A Estrada, Pontevedra). (La hacha de tipo "Bujões-Barcelos" mede de largo $156 \mathrm{~mm})$.

Fig. 9. Codesesa (A Estrada, Pontevedra) metal hoard. (The axe of "Bujóes-Barcelos" type is $162 \mathrm{~mm}$ long).

\section{Caracterización Previa de la Primera Metalurgia del Bronce del noroeste Peninsular}

Se ha considerado que el noroeste de la Península Ibérica fue una importante fuente de recursos de estaño en la antigüedad. El peso de este aspecto en los estudios sobre la prehistoria de la Península Ibérica ha sido muy importante, porque una causa - la metalurgia - y un argumento - la búsqueda de metal - han servido como respuesta a diversos fenómenos de la Prehistoria Reciente. Los aspectos relativos a esta cuestión se desarrollan en detalle en B. Comendador (1999 y 2005) y otros trabajos desarrollados en colaboración (DELIBES \& MONTERO 1999), poniendo

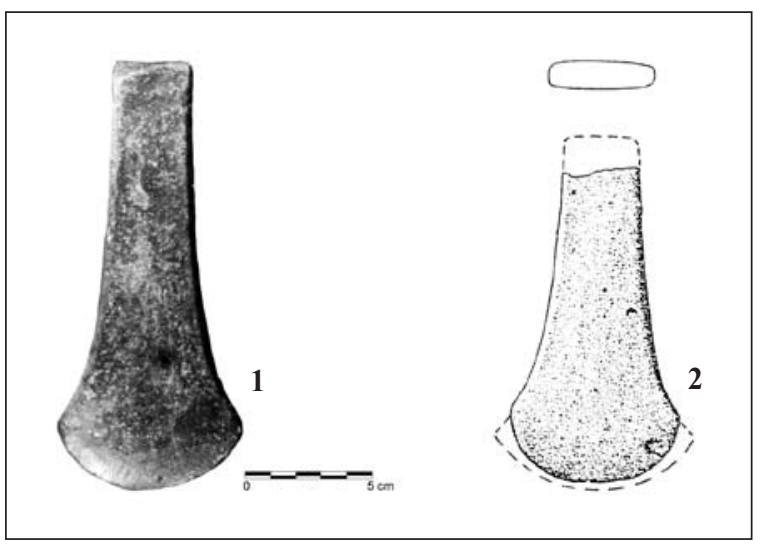

Fig. 10. 1 - Hacha Santa Mariña de Anxeriz (Tordoia, A Coruña); 2 - Hacha de la mina Folgadoura, Viana do Castelo (Monteagudo 1977). (Mede de largo $161 \mathrm{~mm}$ ).

Fig. 10. 1 - Axe from Santa Marina Anxeriz (Tordoia, A Coruña). 2 - Axe from Folgadoura mine, Viana do Castelo (Monteagudo 1977). (The axe is $161 \mathrm{~mm}$ long).

de manifiesto la importancia de la arqueometría en la resolución de problemas específicos, como por ejemplo, la composición de la aleación, especialmente el tanto por ciento en estaño y como las variaciones en el tiempo reflejan una evolución en la tecnología de producción del bronce (COMENDADOR 2005). La determinación específica de la aleación se ha convertido en este momento en una cuestión interesante dentro de la caracterización de los ámbitos tecnológicos de la producción del bronce, desde la caracterización de las primeras producciones a su posterior desarrollo.

Entre los años 60-90, la caracterización del primer bronce se realizó fundamentalmente a partir de la composición química de objetos manufacturados, dada la inexistencia de elementos del proceso de producción. 
El proyecto de análisis sistemáticos más importante fue el publicado en la serie Studien zu der Anfängen der Metallurgie, más conocido por las siglas SAM 9 . Los análisis se realizaron sobre muestras extraídas de los objetos arqueológicos y se analizaron por espectrometría de emisión. Basándose en las composiciones proporcionadas por esa serie analítica se caracterizó la primera producción de bronce del noroeste peninsular por el predominio de aleaciones binarias $\mathrm{Cu}+\mathrm{Sn}$, con un contenido en estaño próximo al ideal eutéctico, en torno al $10 \%$. Porcentajes superiores en estaño se consideraban propios de las aleaciones del Bronce Final (SiERrA et al. 1984; SUÁrEZ 2000).

A partir de los años 90, el programa de análisis sistemáticos Arqueometalurgia de la Península Ibérica integrará nuevos resultados (ROVIRA et al. 1997; DELIBES \& MONTERo 1999; ROVIRA \& GÓMEZ 2003). En este caso, la caracterización del bronce no sólo se hace a partir de objetos acabados, sino que se integran elementos de toda la cadena de producción. El programa integró esencialmente dos vías analíticas; por un lado los análisis no destructivos mediante Energía Dispersiva-Fluorescencia de Rayos X (FRX) y por otro lado, la observación de la estructura del metal mediante el estudio de micrografías.

Dentro del proyectos se analizaron dos gotas y una barra de bronce del yacimiento de la Edad del Bronce de Sola (Nivel IIb) (Braga, Portugal), y dos barras metálicas alargadas de bronce semejantes a punzones de gran tamaño, del islote de Guidoiro Areoso (Illa de Arousa, Pontevedra), ambos considerados entre los objetos de bronce más tempranos de la Península Ibérica (Rovira \& GóMEZ 1994; Co-
MENDADOR 1998; ROVIRA \& MONTERO 2003: nota 2). Las concentraciones de estaño medidas mediante ED-FRX fueron relativamente altas en la mayoría de análisis mostrando porcentajes superiores al $20 \%$ con una pequeña cantidad de elementos traza considerados impurezas (As, Sb...) (Tabla 1).

La observación metalográfica indicó además que uno de los artefactos de Guidoiro sufrió un proceso de homogeneización del metal a través del recocido, una práctica escasa en la metalurgia del Calcolítico y Bronce Antiguo.

A pesar del limitado número de análisis, con base en los resultados del proyecto anterior ${ }^{10}$, las autoras de este trabajo en su momento habían defendido por separado y conjuntamente (BETTENCOURT \& COMENDADOR 2004) la siguiente hipótesis: que los altos valores de estaño en la aleación no son exclusivos de la metalurgia del Bronce Final y que la producción de bronce más temprana se caracterizaría por:

- Altos contenidos en estaño o bronce ricos $(\mathrm{Sn}$ $>15 \%$ );

- Presencia de arsénico y otras impurezas en la aleación;

- Uso de tratamientos mecánicos y aplicación del recocido para la homogeneización de la estructura del metal;

- Falta de control sobre el resultado final. Para esta producción temprana, se proponía que la ausencia de control en el producto final podría resultar de obtener la aleación de bronce reduciendo conjuntamente minerales de cobre y de estaño, incluso un eventual uso de minerales con aleaciones naturales $\mathrm{Cu}-\mathrm{Sn}^{11}$.

Tabla 1. Resultados obtenidos mediante Energía Dispensiva - Fluorescencia de Rayos X (FRX).

Table 1. Energy Dispenser - X-Ray Fluorescence (XRF) results.

\begin{tabular}{|l|l|c|c|c|c|c|c|c|c|c|c|}
\hline \multicolumn{2}{|c|}{} & Fe & Ni & $\mathbf{C u}$ & $\mathbf{Z n}$ & As & Ag & Sn & Sb & Bi & Pb \\
\hline Guidoiro & PA2491 & & & 75,12 & & 1,33 & & 21,52 & & & \\
\hline Guidoiro & & 0.323 & 0.314 & 73.03 & & 1.841 & 0.124 & 21.86 & 1.952 & & 0.011 \\
\hline Guidoiro & $\begin{array}{l}\text { PA4206P } \\
\text { (Pátina) }\end{array}$ & 1.145 & 0.217 & 50.54 & ND & 2.443 & 0.056 & 42.27 & 3.097 & & TR \\
\hline $\begin{array}{l}\text { Sola II } \\
\text { Braga }\end{array}$ & PA6973 & 0.676 & 0.394 & 68.73 & - & 3.989 & - & 26.16 & 0.135 & - & - \\
\hline $\begin{array}{l}\text { Sola II } \\
\text { Braga }\end{array}$ & PA6971 & 0.432 & 0.550 & 69.72 & - & 0.653 & 0.073 & 28.49 & 0.0079 & - & - \\
\hline $\begin{array}{l}\text { Sola II } \\
\text { Braga }\end{array}$ & PA6972 & 0.424 & 0.487 & 72.12 & - & 1.541 & 0.021 & 25.14 & 0.213 & - & - \\
\hline
\end{tabular}

9 Para una revisión general y valoración crítica de la investigación arqueometalúrgica en Galicia consultar X.L. Armada et al. (2008).

10 Incluyendo los resultadosde los análisis del hacha de Nogueira (23,13 Sn) y el puñal de Mesa de Montes (24,75\% Sn), el punzón de la Bauma del Serrat ( $>23 \%$ Sn), o el hacha de Los Fitos (La Cobertoria, Asturias) (18\% Sn) (RoviRA et al. 1997). Fueron realizados eliminando la pátina por abrasión y buscando el núcleo metálico de los objetos. Los valores de Sn resultaban altos en comparación con otros analizados dentro del mismo proyecto y con los mismos parámetros.

11 Aspecto este ya propuesto por S. Rovira y I. Montero (2003). 


\section{Aplicación de la fuente de RAdiación SinCROTRÓNica (SRS) Y LA FUENTE de Dispersión de Neutrones Time- -OF-FLight (TOF) A LA CUESTIÓN DE la Caracterización de la Primera ProducCión de Bronce del Noroeste Peninsular}

El contexto de la aplicación fue la misión científica de corta duración Estaño y Bronce en la Edad del Bronce del Noroeste de la Península Ibérica ${ }^{12}$ integrada dentro del Grupo de trabajo WG1/3 (Procedencia y autentificación) de la acción COST G8 ${ }^{13}$, siendo su principal el de incrementar el conocimiento sobre objetos depositados en museos mediante la aplicación de analíticas no destructivas, para alcanzar una mejor conservación y preservación del patrimonio cultural, así como la promoción de una sinergia entre historiadores, arqueólogos, conservadores y científicos de ciencias naturales (BIDAUD et al. 2006).

Entre los objetivos de la misión corta estaba el de conocer las posibilidades de la aplicación en la investigación sobre metalurgia prehistórica de los siguientes métodos:

- Fuente de Radiación Sincrotrónica (SRS) (Daresbury Lab., Warrington, UK)

- Fuente de dispersión de neutrones Time-of-flight (TOF) (ISIS-ROTAX, Rutherford-Appleton Labs., Didcot, UK).

El equipo utilizado para la realización de la difracción de neutrones (TOF) en bronce y en otros materiales ha sido descrito previamente (PANTOS et al. 2005; KOCKELMANN et al. 2000; SiANO et al. 2002; SIANO Y KOCKELMANN 2003). La herramienta ROTAX en ISIS en el laboratorio Rutherford Appleton utiliza la técnica time-of-flight, que, como su propio nombre indica, determina las energías o velocidades de los neutrones midiendo sus tiempos de vuelo (KocKeLMANN et al. 2004).

En arqueología, los análisis mediante difracción de Rayos X casi siempre requieren la extracción de una muestra y normalmente, con intención de dañar lo menos posible al objeto, se toma una muestra pequeña que es poco representativa de la totalidad del objeto. En este sentido, la difracción de neutrones es singular porque debido al alto poder de penetración de los neutrones, es posible estudiar piezas enteras que pueden ser de gran tamaño y no es necesario toma de muestra. Los neutrones penetran en el in- terior del objeto y proporcionan información, ya de la superficie, ya del interior. Para las mediciones en el difractómetro ROTAX en ISIS, las muestras fueron iluminadas con un haz de sección cruciforme de 20 x $20 \mathrm{~mm}$. Así pues los datos de la difracción contienen un promedio de la totalidad del objeto iluminado. Cada medición puede ser representativa de la totalidad, pero como además la analítica no es destructiva, se pueden hacer varias mediciones para contrastarlas. La difracción de neutrones permite una identificación de las fases cristalinas a través del área examinada, incluyendo información a cerca de las fases presentes en la aleación y las fases de la corrosión en la superficie del objeto, así como información estructural.

Procediendo a comentar los resultados de la aplicación, la difracción mediante fuente de Radiación Sincrotrónica (SRS) posibilitó una identificación muy pormenorizada de los elementos de corrosión, siendo estos resultados de gran utilidad en el campo de la restauración, porque permiten diseñar los métodos de actuación más adecuados para la conservación del metal (COMENDADOR et al. 2008; CoMENDADOR Y MÉNDEZ 2006). Sin embargo, este tipo de análisis se mostró poco operativo en el campo de la arqueometalurgia.

La figura 11 y la tabla 2 muestran los patrones de difracción de neutrones y los objetos de bronce de los que fueron obtenidos los datos: fragmentos de los punzones de Guidoiro; las piezas de Sola y la gota de fundición de Bouça da Cova da Moura.

De modo general se observa que la concentración de los valores de estaño de estos objetos está entre el $5-10 \%$ en cuanto a la masa de la aleación (fase alfa), no siendo descartable en algunos ejemplos la presencia de fases de aleación ricas en estaño.

Estos valores de estaño resultan menores que los obtenidos mediante ED-FRX (Tabla 3), pero la comparación de los resultados proporcionados no es fácil, porque la difracción de neutrones analiza fases cristalinas, mientras que la Fluorescencia de Rayos X identifica elementos. En todo caso, la difracción de neutrones pone de manifiesto algunas limitaciones la metodología ED-FRX cuando se trata del análisis de aleaciones, ya que para determinar la composición es necesario estudiar secciones pulidas donde quede expuesto el interior no corroído del objeto. En ocasiones, cuando los objetos de bronce están muy corroídos, no pueden pulir y limpiar apropiadamente y se corre el riesgo de sobrevalorar

12 Acción realizada por Beatriz Comendador bajo la supervisión de Susana Reboreda (Universidad de Vigo). Los investigadores implicados fueron Emmanuel Pantos (Daresbury Labs.) y Winfried Kockelmann (Rutherford-Appleton Labs.). El proyecto contó con el asesoramiento de Ignacio Montero (CSIC).

13 Para más información sobre el programa científico de la ya finalizada acción vid. http://srs.dl.ac.uk/arch/cost-g8. 


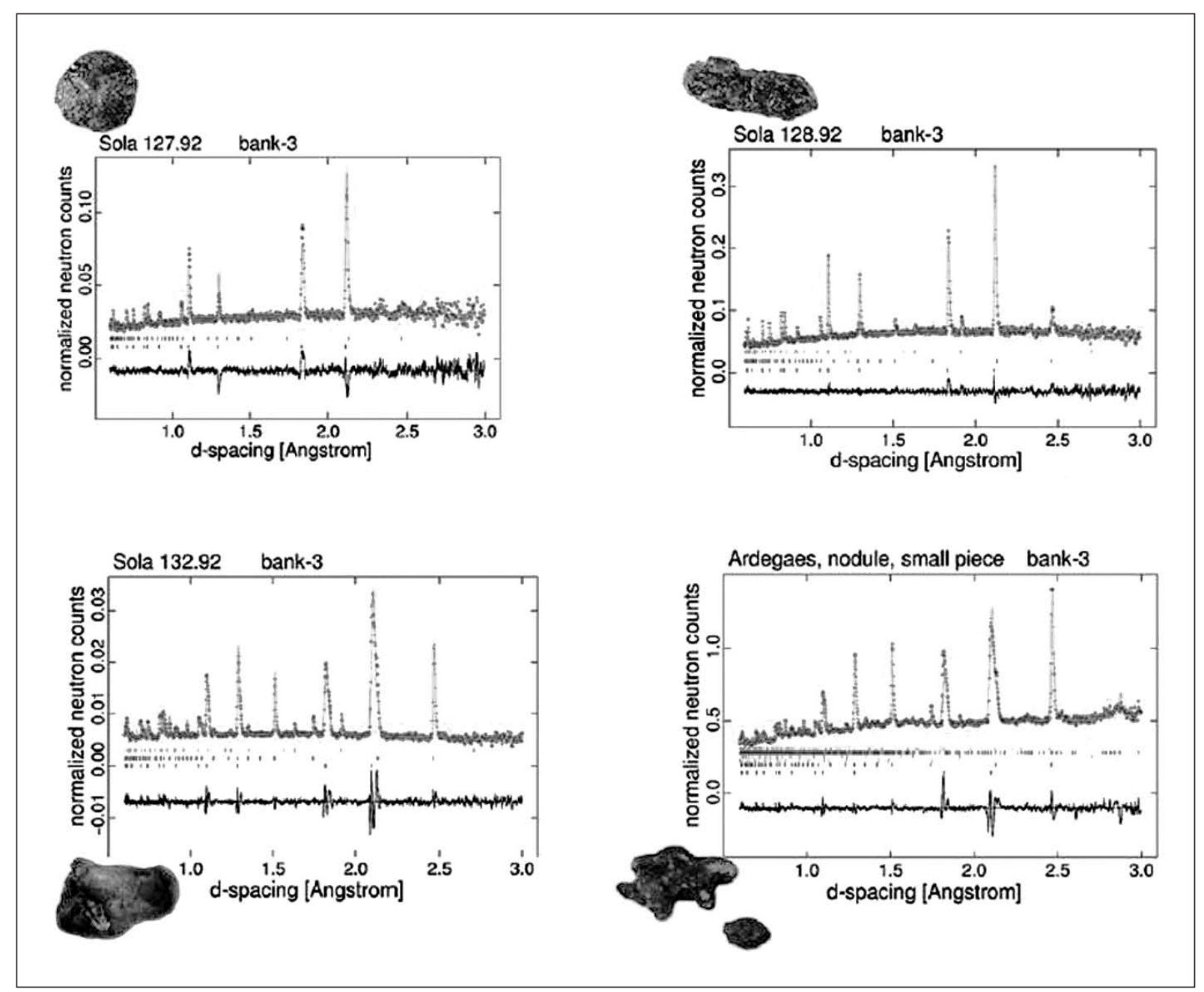

Fig. 11. Patrones de la difracción de neutrones tomados en ROTAX.

Fig. 11. Neutron diffraction patterns taken from ROTAX.

Tabla 2. Resultados obtenidos mediante la difracción de neutrones.

Table 2. Neutron diffraction results.

\begin{tabular}{|c|c|c|c|}
\hline $\begin{array}{l}\text { Bouça da Cova da } \\
\text { Moura (grande) }\end{array}$ & $\begin{array}{l}95 / 5 \% \mathrm{Cu} / \mathrm{Sn} \text { bronce } \\
\text { (fase alfa) }\end{array}$ & $\begin{array}{l}\text { + cuprita, nantoquita, } \\
\text { malquita y cuarzo }\end{array}$ & $\begin{array}{l}\text { La distribución de estaño no es homogénea. No se puede } \\
\text { excluir la presencia de fases delta ricas en estaño }\end{array}$ \\
\hline $\begin{array}{l}\text { Bouça da Cova da } \\
\text { Moura (pequeño) }\end{array}$ & $\begin{array}{l}95 / 5 \% \mathrm{Cu} / \mathrm{Sn} \text { bronce } \\
\text { (fase alfa) }\end{array}$ & $\begin{array}{l}\text { + cuprita, nantoquita, } \\
\text { cassiteerita y malaquita }\end{array}$ & $\begin{array}{l}\text { La distribución de estaño no es homogénea. No se puede } \\
\text { excluir la presencia de fases delta y eta ricas en estaño }\end{array}$ \\
\hline $\begin{array}{l}\text { Sola } 127.92 \\
\text { Resto }\end{array}$ & $\begin{array}{l}90 / 10 \% \mathrm{Cu} / \mathrm{Sn} \text { bronce } \\
\text { (fase alfa) }\end{array}$ & + cuprita & $\begin{array}{l}\text { Los picos sugieren que fue sometida a un proceso de } \\
\text { homogeneización. No se puede excluir la presencia de } \\
\text { fases delta y eta ricas en estaño }\end{array}$ \\
\hline $\begin{array}{l}\text { Sola } 128.92 \\
\text { Vareta }\end{array}$ & $\begin{array}{l}90 / 10 \% \mathrm{Cu} / \mathrm{Sn} \text { bronce } \\
\text { (fase alfa) }\end{array}$ & + cuprita & $\begin{array}{l}\text { Los picos sugieren que fue sometida a un proceso de } \\
\text { homogeneización. Se puede excluir la presencia de fases } \\
\text { ricas en estaño }\end{array}$ \\
\hline Sola 132.92 & $\begin{array}{l}95 / 5 \% \mathrm{Cu} / \mathrm{Sn} \text { bronce } \\
\text { (fase alfa) }\end{array}$ & + cuprita, nantoquita & $\begin{array}{l}\text { La distribución de estaño no es homogénea. No se puede } \\
\text { excluir la presencia de fases delta y eta ricas en estaño }\end{array}$ \\
\hline
\end{tabular}


Tabla 3. Comparación de los resultados obtenidos mediante ED-FRX y TOF.

Table 3. Comparison of the results obtained through EDXRF and TOF.

\begin{tabular}{|l|l|l|}
\hline Procedencia & $\begin{array}{l}\text { Programa Arqu. } \\
\text { (ED-FRX) 1995 }\end{array}$ & $\begin{array}{l}\text { ISIS } \\
\text { (TOF) 2005 }\end{array}$ \\
\hline Sola & PA6973: Sn 26.16\% & Sn 10\% \\
\hline Sola & PA6971: Sn 28.49\% & Sn 10\% \\
\hline Sola & PA6972: Sn 25.14\% & Sn 5\% \\
\hline Guidoiro & PA2491: Sn 21,52\% & $\begin{array}{l}\text { Sn (muestra no } \\
\text { significativa) }\end{array}$ \\
\hline
\end{tabular}

Posteriormente a la redacción original de este trabajo, en el 2008, recibimos los resultados de un nuevo análisis del fragmento del punzón de Guidoiro, realizado en otro difractómetro de neutrones (ISIS/GEM). El resultado es: cuprita $=84,9 \%$; atacamita $=4,2 \%$; paratacamita $=0.6 \%$; malaquita $=0.7 \%$; cuarzo $=2.5$ y casiterita $=7.1 \%$. Agradecemos su interés en este trabajo al Dr. Winfried Kockelmann del Rutherford Appelton Laboratory.

Following the initial drafting of this study, in 2008, we received the results of a new analysis of the Guidoiro awl fragment, from another neutron diffractometer (ISIS/GEM). The results are: cuprite $=84.9 \%$; atacamite $=4.2 \%$; paratacamite $=0.6 \%$; malachite $=0.7 \%$; quartz $=2.5 \%$ and cassiterite $=7.1 \%$. We are very grateful to Dr. Winfried Kockelmann of Rutherford Appleton Laboratory for his interest in this work.

el estaño que suelen contener las pátinas en mayor cantidad. Idealmente las muestras deben presentar una superficie plana y pulida, pero hay corrosión intergranular difícil de eliminar salvo que se haga un pulido en profundidad, lo que no es aceptable en la analítica de material arqueológico. Este podría ser el caso del análisis de los punzones de Guidoiro ${ }^{14}$.

Como ha señalado E. Figueiredo (2004), la penetración del haz incidente de Rayos X es muy pequeña en el análisis de materiales metálicos, por lo que los tenores de elementos analizados pueden no corresponder a los tenores de la aleación original. Factores tales como el área y la superficie analizadas son importantes. E. Figueiredo (2004) indica que diversos fenómenos pueden provocar diferencias entre la composición de la aleación en el interior y en la superficie: fenómenos de corrosión como la lixiviación preferencial de determinados compuestos, tales como los de cobre; incorporación de elementos provenientes de los suelos de inhumación; segregación preferencial en determinado elemento durante la solidificación del artefacto, para la super- ficie en el caso del estaño; desplazamiento mecánico de una fase de menor temperatura de fusión durante un proceso de calentamiento con martillado, etc. Deduce que los datos cuantificados por $F R X$ resultan en tenores de $\mathrm{Sn}$ y Fe por encima de los existentes en la aleación. A través de micro-fluorescencia de Rayos X verifica la existencia de enriquecimiento superficial en determinados elementos como el estaño, que está presente en la pátina de los artefactos de bronce en valores que pueden ser cuatro veces superiores al contenido de la aleación original, por lo que recomienda interpretar con prudencia los valores cuantificados por FRX.

La difracción de neutrones, por su parte, permite una determinación precisa y no destructiva de los porcentajes de la aleación de las fases cobre-estaño, incluso en presencia de capas de corrosión. Pero en síntesis, no se pueden obtener conclusiones firmes en relación con este aspecto a partir del reducido número de objetos analizados en este trabajo.

En cuanto a la concentración de elementos traza, es otro parámetro importante que actualmente puede obtenerse mediante una técnica de neutrones diferente (Prompt Gamma Activation Análisis-PGAA), no disponible entre los equipos utilizados en ISIS.

Por último, en cuanto a información estructural, el resultado de los análisis de los objetos Sola 127.92 y Sola 128.92 sugiere que ambos sufrieron un proceso de homogeneización térmica.

\section{Consideraciones Finales}

Frente a los resultados y problemáticas expuestas, podemos extraer las siguientes conclusiones:

1) la arqueometría es fundamental para la resolución de problemas específicos asociados a la composición de las aleaciones y a su variación a lo largo del tiempo;

2) la aplicación de esta nueva metodología (difracción de neutrones (TOF) es bastante fiable en el estudio de los bronces muy corroídos;

3) a pesar de la pequeña muestra, la nueva metodología analítica muestra aleaciones binarias pobres de o de buena calidad $( \pm 10 \% \mathrm{Sn})$ para el noroeste peninsular;

4) estos resultados son concordantes con los obtenidos para los materiales localizados en Fraga dos Corvos ${ }^{15}$;

14 En relación con el análisis de uno de los punzones de Guidoiro, S. Rovira ya indicó que la cantidad de bronce sería en torno al $10 \%$ según la metalografía, señalando este método como más fiable que el análisis por fluorescencia de rayos x previamente realizado. Remarca además en la metalografía la ausencia de segregados de fase delta, que se producen en las microestructuras de recocido cuando el porcentaje de estaño es superior al 13-14\% (ROVIRA Y GÓMEZ 2003 y comunicación personal de S. RovIRA, Abril de 2000).

15 Comunicación personal de J.C. Senna-Martinez. 
5) tales consideraciones nos permiten poner en duda la hipótesis expuesta por nosotras anteriormente, concretamente en lo referido a los altos contenidos de estaño ( $\mathrm{Sn}>15 \%)$ en los bronces tempranos del noroeste;

6) mientras tanto, sólo el desarrollo de nuevas analíticas podrá contribuir a la resolución de esta problemática;

7) dado que el poblado de Sola fue destruido, las estratigrafías de Guidoiro e Fixón - Costa da Seixeira son problemáticas o desconocidas, la continuidad de los trabajos en Bouça da Cova da Moura se torna fundamental para aumentar el conocimiento sobre la primera metalurgia del bronce, sobre todo en la fachada más occidental del noroeste de la Península Ibérica.

\section{Agradecimientos}

Las autoras agradecen a los Laboratorios de Daresbury la financiación de la participación de B. Comendador Rey en el COST-G8 Máster Class on SR in Cultural Heritage, en Octubre de 2005, con la STSM (Short Term Scientific Mission): Tin and Bronze in the Bronze Age of the North-West Iberian Peninsula (COST G8 grupo de trabajo: WG1/3), a Susana Reboreda (de la Universidade de Vigo), a Emmanuel Pantos, Anthony Bell y Mike Macdonald (del CCLRC, Daresbury Laboratory, Warrington), a Winfried Kockelmann (del ISIS, Rutherford-Appleton Laboratory, Didcot), a Salvador Rovira e a Ignacio Montero (del C.S.I.C.), a Panagiota Manti (del Conservation Department, Cardiff University), a António Monge Soares (del Instituto Tecnológico e Nuclear (ITN), a João Carlos Senna-Martinez (de la Universidade de Lisboa), a Pedro Pimenta (de la Universidade do Minho), a André T. Ribeiro (de la Câmara Municipal da Maia), a Andreia Arezes, arqueóloga y a Jorge Guedes, arqueólogo.

La publicación de este trabajo debe encuadrarse igualmente en el ámbito del Proyecto Metalurgia Primitiva no Território Português - EARLYMETAL (PTDC/HIS-ARQ/ /110442/2008), financiado por la Fundação para a Ciência e a Tecnologia (FCT) de la cual B.C.R. es asesora y A.M.S.B. es investigadora.

\section{BIBLIOGRAFIA}

AlmEIDA, C.A.B. 1988. Carta arqueológica do concelho de Esposende. Boletim Cultural de Esposende 13/14: 21-44.

AlmeidA, P.B. \& Fernandes, F. 2008. O povoado da Idade do Bronze da Cimalha. Actas do I Encontro de Arqueologia das Terras do Sousa. Oppidum n. ${ }^{\circ}$ especial. Lousada: Câmara Municipal: 29-44.

Alonso, F. \& Bello, J.M. ${ }^{\text {a }}$ 1997. Cronología de la periodización del fenómeno megalítico en Galicia a la luz de las dataciones por Carbono14. In A. Rodriguez CASAL (ed.) O Neolítico atlántico e as orixes do megalitismo. Actas do Coloquio internacional 1996. Santiago de Compostela: Universidad de Santiago de Compostela: 507-520.
Armada, X.L.; Comendador, B. \& García Vuelta, O. 2008. La investigación arqueométrica sobre la metalurgia en Galicia: una aproximación a su trayectoria y estado actual. In S. RoVIRA; M. GARCíA-Heras; M.G. MORET \& I. MONTERO RUIZ (eds.) Actas do VII Congreso de Ibérico de Arqueometría, Madrid, 8-10 de Octubre 2007. Madrid: Lámina: 410-431.

BetTenCOURT, A.M.S. 1997. Expressões funerárias da Idade do Bronze no Noroeste peninsular. Actas do II. ${ }^{\circ}$ Congreso de Arqueología Peninsular (Zamora, 2006). Vol. 2. Zamora: Fundación Rei Afonso Henriques: 621-632.

BETTENCOURT, A.M.S. 1999. A paisagem e o homem na bacia do Cávado durante o II e o I milénios AC. 5 vols. Braga (Tesis doctoral presentada en la Universidade do Minho).

Bettencourt, A.M.S. 2000. O Povoado da Idade do Bronze da Sola, Braga, Norte de Portugal. Cadernos de Arqueologia Monografias 9. Braga: Unidade de Arqueologia da Universidade do Minho.

Bettencourt, A.M.S. 2001. Aspectos da metalurgia do bronze no Entre-Douro-e-Minho no quadro da Proto-História do Noroeste Peninsular. Arqueologia 26: 13-40.

BetTenCOURT, A.M.S. 2010a. Estruturas e práticas funerárias do Bronze Inicial e Médio do Noroeste Peninsular. In P. BUENO, A. Gilman, C.M. Morales \& F.J. SÁnCheZ-Palencia (eds.) Arqueología, Sociedad, Territorio y Paisaje. Estudios sobre Prehistoria Reciente, Protohistoria y Transición al Mundo Romano en Homenaje a M. ${ }^{a}$ Dolores Fernández Posse. Bibliotheca Praehistorica Hispana (BPH) 27. Madrid: CSIC: 115-139.

Bettencourt, A.M.S. 2010b. Comunidades pré-históricas da bacia do Leça. In J. VARElA \& C. PIRES (coords.) O Rio da Memória: Arqueologia no Território do Leça. Matosinhos: Câmara Municipal: 33-88.

Bettencourt, A.M.S. \& Comendador, B. 2004. Los inicios de la metalurgia del bronce en el Noroeste Peninsular. In J. MATA (ed.) Actas do IV Congreso Internacional sobre Patrimonio Geológico y Minero (Teruel, Setembro 2003). Teruel: Museu do Instituto Geológico e Mineiro de Utrillas: 343-357.

Bettencourt, A.M.S.; Dinis, A.; Figueiral, I.; Rodrigues, A.; Cruz, C.; Silva, I.S.; Azevedo, M. \& Barbosa, R. 2007. A ocupação do território e a exploração de recursos durante a Pré-História Recente do Noroeste de Portugal. In S.O. JorGE, A.M.S. Bettencourt \& I. Figueiral (eds.) A Concepção das Paisagens e dos Espaços na Arqueologia da Península Ibérica. Actas do IV Congresso de Arqueologia Peninsular. Faro: Centro de Estudos de Património / Departamento de História, Arqueologia e Património da Faculdade de Ciências Humanas e Sociais da Universidade do Algarve: 149-164.

Bettencourt, A.M.S.; Alves, L.B.; Ribeiro, A.T. \& Menezes, R.T. en prensa. Gravuras rupestres da Bouça da Cova da Moura (Ardegães, Maia, Norte de Portugal), no contexto da Pré-História Recente da bacia do Leça. Gallaecia 31.

Bidaud, E.; Benedetti, D.; Bugoi, R.; Comendador, B.; Goidanich, S.; Gutierrez, C.; Garipoli, D.; Manti, P.; Mifsud, T.; Urbina, V.; Kockelmann, W.; Smith, A.D.; Bahrami, F.; Macdonald, M.A.; Bell, A.T.; Jones, R.L.; Rizkallah, P. \& Pantos, E. 2006. The COST-G8 master class on SR in cultural heritage at DL-SRS and RAL-ISIS. Proceedings of SR2A06 - Synchrotron Radiation in Art and Archaeology Conference (Berlin 27-30 September 2006). OnLine Hyperlink http://srs.dl.ac.uk/arch/Posters/E.Bidaud-etalSR2A06-Berlinslides_files

Blas CortinA, M.A. de 1983. La Prehistoria Reciente de Asturias Oviedo: Fundación Pública de Cuevas y Yacimientos Prehistóricos de Asturias.

CAstro Pérez, L. 1997. Brazalete de bronce de Santo Tirso. Santo Tirso Arqueológico 1. 2. ${ }^{\mathrm{a}}$ série: 5-11. 
COMENDAdOR, B. 1998. Los inicios de la metalurgia en el Noroeste de la Península Ibérica. Brigantium - 11. A Coruña: Museo Arqueolóxico do Castelo de San Antón.

COMENDADOR, B. 1999a. The early development of metallurgy in the North-West of the Iberian Peninsula. In S.M.M. YounG; A.M. POllard; P. BUdD \& R.A. IXER (eds.) Metals in Antiquity. Proceedings of the International Conference Metals in Antiquity (Boston, EE.UU., 1997). BAR International Series 792. Oxford: Archaeopress: 516-537.

Comendador, B. 1999b. Cambios de escala en la producción metalúrgica durante las fases finales de la edad del bronce en el Noroeste peninsular. Actas do Congresso de Proto-História Europeia (Guimarães 1999). Revista de Guimarães n. ${ }^{\circ}$ especial. Guimarães: Sociedade Martins Sarmento: 515-537.

Comendador, B. 2005. Beyond the Cassiterides: tin and bronze in the Bronze Age of the North-West of the Iberian Peninsula. Proceedings of the Archaeometallurgy Conference (Londres, 29-30 Abril 2005). On-Line Hyperlink http://webs.uvigo.es/ beacomendador/index_archivos/POSTER.pdf

COMENDADOR, B. 2007. Bajando a la mina: una fugaz visita a la minería prehistórica (1. ${ }^{\mathrm{a}}$ parte). Revista da Asociación de Amigos do Arqueolóxico 12: 22-32.

Comendador, B. \& MÉndez, J.L. 2006. A Patina over time: ancient metals conservation in North-Western Iberia. Actas del Simposium Looking Forward For The Past: Science An Heritage(London, 28 Noviembre 2006). On-Line Hyperlink http://www.srs.ac.uk/scienceandheritage/posters.htm

Comendador, B. \& Bettencourt, A.M.S. 2007. Novos dados sobre a primeira metalurgia de bronze no Noroeste Peninsular: os contributos da bacia do Leça. I Jornadas Arqueológicas da Bacia do Rio Leça, Matosinhos (Outubro, 2007). On-Line Hyperlink http://webs.uvigo.es/beacomendador/index_archivos/Jornadas.pps

Comendador, B. \& Méndez, L. 2007. ¿Patrimonio o Chatarra?: Arqueología experimental y metal. In M. RAMOS, J.E. GONZÁlez \& J. BAENA (eds.) Arqueología Experimental en la Península Ibérica. Investigación, didáctica y patrimonio. Actas I Congreso de Arqueología Experimental (Santander, 2005). Santander: Asociación Española de Arqueología Experimental: 317-328.

Comendador, B.; Reboreda, S.; Kockelmann, W.; MacDoNALD, M.; BELL, T. \& PANTOS, M. 2008. The sources of tin: early bronzes technology and the route to the land's end in North-Western Iberia. In S.A. PAIPETIS (ed.) Science and technology in Homeric Epics. History of Mechanism and Machine Science 6: Springer Verlag. On-Line Hyperlink http://srs.dl.ac. uk/arch/Posters/Commendador-Olympia06-10S_files/frame. htm

Coffyn, A. 1985. Le bronze final atlantique dans la Péninsule Ibériques. Paris: Diffusion du Boccard.

CORTEZ, F.R. 1946. Machados e outros objectos de bronze. Porto: Museu Nacional de Soares dos Reis.

Delibes, G. \& Montero, I. (eds.) 1999. Las primeras etapas metalúrgicas en la Península Ibérica II. Estudios Regionales. Madrid: Instituto Universitario Ortega y Gasset.

FÁbregas Valcarce, R.; Bonilla Rodríguez, A. \& CÉsar VIla, M. 2007. Monte dos Remédios (Moaña, Pontevedra). Un asentamiento de la Prehistoria Reciente. Polígono del Tambre: Tórculo Edicións.

FigueIREDO, E. 2004. A espectrometria de fluorescência de raios $X$ na caracterização química de um conjunto de artefactos metálicos pré-históricos do Castro de Pragança. Lisboa (Trabajo de Licenciatura presentado en la Universidade de Lisboa, inédito - policopiado).

Figueiredo, E. \& ARAújo, M. ${ }^{\mathrm{a} F}$. 2005. Influence of corrosion layers in EDFRX analysis of buried copper based prehistoric artefacts. European Corrosion Congress Proceedings. Lisboa: cd-rom 0-481-4.

Geirinhas, F.; Gaspar, M.; SENNA-MARTINEZ J.C.; Figueiredo, E.; AraúJo, M.F. \& Silva R.J.C. 2011. Copper isotopes on artifacts from Fraga dos Corvos First Bronze Age habitat site and nearby $\mathrm{Cu}$ occurrences: an approach on metal provenance. In J.M. MATA-PERElló, L. TORRo I ABAT \& M.N. FuenteS Prieto (eds.) A.N. CAMPO \& O. PUCHA Riart (co-eds.) Actas del Quinto Congreso Internacional sobre Mineria y Metalurgia Históricas en el Suroeste Europeu (Léon, 2008). Libro en Homenaje a Claude Domergue. Léon: SeDPGYM: 177-184.

Jorge, S.O. 1986. Povoados da Pré-História Recente (III. ${ }^{\circ}$ inícios do II. ${ }^{o}$ milénios a.C.) da região de Chaves e $V^{a}{ }^{a} .^{a}$ de Aguiar (Trás-os-Montes Ocidental). Porto: Instituto de Arqueologia da Faculdade de Letras. 3 vols.

JoRge, S.O. 1988. O povoado da Bouça do Frade (Baião) no quadro do Bronze Final do Norte de Portugal. Monografias Arqueológicas do GEAP 2. Porto: Grupo de Estudos Arqueológicos do Porto.

Kockelmann W.; Pantos, A. \& Kirfel, A. 2000. Neutron and synchrotron radiation studies of archaeological objects. In D.C. CReagh \& D.A. BRADley (eds.) Radiation in Art and Archaeometry. Amsterdam: Elsevier Science: 347-377.

Kockelmann W.; Kirfel, A.; Siano, S. \& Frost, C. 2004. Illuminating the past: the neutron as a tool in archaeology. Physics Education 39 (2): 155-165.

Monteagudo, L. 1954. Metalurgia hispana de la Edad del Bronce, con especial estudio de Galicia y Norte de Portugal. Cesaraugusta 4: 55-95.

Monteagudo, L. 1977. Die beile auf der Iberischen Halbinsel. Prahistorische Bronzefunde 9 (6). Munich.

Pantos, E.; Kockelmann, W.; Chapon, L.C.; Lutteroti, L.; Bennet, S.L.; Tobin, M.J.; Mosselmans, J.W.F.; Pradell, T.; Salvadó, N.; Buti, S.; Garer, R. \& PraG, J.N. 2005. Neutron and $\mathrm{x}$-ray characterisation of the metallurgical properties of a $7^{\text {th }}$ century BC Corinthian-type bronze helmet. Nucl. Instrum. Methods B 239:16-26.

Reimer, P.J.; BAillie, M.G.L.; BARD, E.; BAYliss, A.; BeCK, J.W.; BlackWell, P.G.; Bronk Ramsey, C.; Buck, C.E.; BurR, G.S.; EDWARDS, R.L.; FrIEDRICH, M.; GROOTES, P.M.; Guilderson, T.P.; Hajdas, I.; Heaton, T.J.; HogG, A.G., Hughen, K.A.; Kaiser, K.F.; Kromer, B.; MCCORMAC, F.G.; MANNING, S.W.; REIMER, R.W.; RichaRdS, D.A.; SOUThON, J.R.; TALAMO, S.; TURNEY, C.S.M.; Plicht, J. VAN Der \& WeYhenMeYer, C.E. 2009. IntCal09 and Marine09 radiocarbon age calibration curves, 0-50,000 years cal BP. Radiocarbon 51: 1111-1150.

Ribeiro, A.T.; Alves, L.B.; Bettencourt, A.M.S. \& Menezes, R.T. 2010. Space of memory and representation: Bouça da Cova da Moura (Ardegães, Maia, Northwest of Portugal) - a case study. In A.M.S. Bettencourt, M.J. SAnCheS, L.B. Alves \& R. FÁBREGAS VALCARCE (eds.) Conceptualizing space and place. On the role of agency, memory and identity in the construction of space from the Upper Paleolithic to the Iron Age in Europe. Proceedings of the 15th Crongress of the International Union for Prehistoric and Protohistoric Sciences, Lisbon, September 2006, BAR-S2058 International Series, Oxford: Archeopress: 89-98.

Rovira, S.; Montero, I. \& Consuegra, S. 1997. Las primeras etapas metalúrgicas en la Península Ibérica. Madrid: Instituto Universitario Ortega y Gasset.

Rovira, S. \& Gomez, P. 1994. Punzones y varillas metálicas en la Prehistoria Reciente española: un estudio tecnológico. Espacio, Tiempo y Forma. Serie I. Prehist. y Arqueol. 7: $371-402$. 
RoviRA, S. \& GOMEZ, P. 2003. Las primeras etapas metalúrgicas en la Península Ibérica. III. Estudios Metalográficos. Madrid: Instituto Universitario Ortega y Gasset.

Rovira, S. \& Montero, I. 2003. Natural tin-Bronze alloy in Iberian Peninsula metallurgy: potentiality and reality. In A. GIUMLIA-MAIR \& F. SCHIAVO (eds.) Le problème de l'étain à l'origine de la métallurgie. BAR International Series 1199. Oxford: Archaeopress: 15-22.

SANCHES, M.J. 1982. Vasos da estação arqueológica de Corvilho - Santo Tirso. Arqueologia 5: 56-61.

Senna-Martínez, J.C. \& Luís, E. 2009. A Fraga dos Corvos (Macedo de Cavaleiros): um sítio de habitat da primeira Idade do Bronze em Trás-os-Montes Oriental. A Campanha 6 (2008). Cadernos Terras Quentes 6: 69-79.

Senna-Martínez, J.C.; Ventura, J.M. \& Carvalho, H.A. 2005. A Fraga dos Corvos (Macedo de Cavaleiros): um sítio de habitat do "Mundo Carrapatas" da primeira Idade do Bronze em Trás-os-Montes Oriental. Cadernos Terras Quentes 2: 61-82.

Senna-Martínez, J.C.; Ventura, J.M.; Carvalho, H.A. \& FiGUEREDO, E. 2006. A Fraga dos Corvos (Macedo de Cavaleiros): um sítio de habitat da primeira Idade do Bronze em Trás-os-Montes Oriental. A Campanha 3. Cadernos Terras Quentes 3: 61-85.

Siano, S.; Kockelmann, W.; Bafile, U.; Celli, M.; Iozzo, M.; Miccio, M.; Moze, O.; Pini, R.; SAlimbeni, R. \& ZopPi, M. 2002. Quantitative multiphase analysis of archaeological bronzes by neutron diffraction. Applied Physics A 74 (Suppl.): S1139-S1142.
Siano, S.; Kockelmann, W.; Bafile U.; Celli M.; Iozzo M.; Miccio M.; DanN J.A. \& Garagnani G. 2003. Microestructural bronze characterisation by time of flight neutron diffraction. Proc. Int. Conf. Archaeometallurgy in Europe (24/26 September 2003). Vol. 2. Milan: Assoziacione Italiana de Metalurgia: 319-329.

Sierra, J.C.; VAZQuerz, A.J.; Luis, L. DE \& FerreirA, S. 1984. El deposito del Bronce Final de Samieira. Boletín Auriense. Anexo 2. Ourense.

Stuiver, M. \& ReIMER, P.J. 1993. Extended 14C Data Base and Revised CALIB 3.0 14C Age Calibration. Radiocarbon 35 (1): $215-230$

SuÁrez, J. 1995. O Fixón: una nueva perspectiva del Bronce Inicial en Galicia. Actas del XXII Congreso Nacional de Arqueología (Vigo, 1993). Vigo: Instituto de Estudios Vigueses: 56-67.

SUÁREZ, J. 2000. Las hachas de talón sin anillas y la introducción del Bronce Atlántico en el No Hispánico. Ourense: Boletín Auriense 30: 9-46.

SuÁrez, J.; Carballo, X. \& Amil, X.C. 1997. Un molde de fundición para machados "tipo Barcelos" procedente do Monte das Carballas: Guillade, Ponteareas (Pontevedra). Soberosum 2: $21-24$.

TeiXeirA, C. \& Fernandes, M.S.C. 1963-1964. Machados planos de Montalegre. Trabalhos de Antropologia e Etnologia 19 (2): 169-173

ZBYZEWSKI, G. \& FERREIRA, O.V. 1955. Sur une plaque anthropomorphe en cuivre trouvée dans la mine d'étain de "Folgadoura". Comunicação dos Serviços Geológicos de Portugal 36: $49-50$ 